\title{
Evaluation of an advanced pressure ulcer management protocol followed by trained wound, ostomy, and continence nurses: a non-randomized controlled trial
}

\author{
This article was published in the following Dove Press journal: \\ Chronic Wound Care Management and Research \\ 24 February 2015 \\ Number of times this article has been viewed
}

\begin{abstract}
Toshiko Kaitani'
Gojiro Nakagami²

Junko Sugama ${ }^{3}$

Masahiro $\mathrm{Tachi}^{4}$

Yutaka Matsuyama ${ }^{5}$

Yoshiki Miyachi ${ }^{6}$

Takashi Nagase ${ }^{2}$

Yukie Takemura ${ }^{7}$

Hiromi Sanada ${ }^{2}$

'School of Nursing, Sapporo

City University, Hokkaido, Japan;

${ }^{2}$ Department of Gerontological

Nursing/Wound Care Management,

Graduate School of Medicine, The

University of Tokyo, Tokyo, Japan;

${ }^{3}$ Department of Clinical Nursing, Institute of Medical, Pharmaceutical and Health Sciences, Kanazawa University, Kanazawa, Japan; ${ }^{4}$ Department of Plastic Surgery, Tohoku University Graduate School of Medicine, Miyagi, Japan; ${ }^{5}$ Department of Biostatistics, School of Public Health, Graduate School of Medicine, The University of Tokyo, Tokyo, Japan; 'Department of Dermatology, Kyoto University Graduate School of Medicine, Kyoto, Japan; 'Department of Nursing, Research Hospital, The Institute of Medical Science, The University of Tokyo, Tokyo, Japan
\end{abstract}

Correspondence: Hiromi Sanada Department of Gerontological Nursing/ Wound Care Management Graduate School of Medicine, The University of Tokyo, Faculty of Medicine Bldg. No.5-306 7-3-I Hongo, Bunkyo-ku,

Tokyo II3-0033, Japan

$\mathrm{Tel}+8|3584| 3419$

Fax +81358413419

Email hsanada-tky@umin.ac.jp

\begin{abstract}
Aims and objectives: We investigated the effectiveness and safety of an advanced pressure ulcer (PU) management protocol comprising 1) ultrasonography to assess the deep tissue, 2) use of a non-contact thermometer to detect critical colonization, 3) conservative sharp debridement, 4) dressing selection, 5) negative pressure wound therapy, and 6) vibration therapy in comparison with those of a conventional approach. Each protocol was followed by trained wound, ostomy, and continence nurses (WOCNs).
\end{abstract}

Background: At present, there is no systematic PU management protocol for nurses that includes appropriate assessment and intervention techniques for deep tissue injury and critical colonization. In Japan, there is no such protocol that the nurses can follow without a physician's orders.

Design and methods: This was a prospective non-randomized controlled trial. Over a 3-week period, we evaluated the effectiveness of an advanced protocol by comparing the PU severity and healing on the basis of the DESIGN-R scale and presence of patients' discomfort. We recruited ten WOCNs to follow the advanced protocol and 19 others as controls. Statistical analysis included a linear mixed-effects model and a logistic regression model.

Results: In week $0-1$, the advanced protocol was significantly associated with prevention of PU deterioration. Using the linear mixed-effects model, we observed a greater decrease in the DESIGN-R score (healing) in the advanced protocol group. There were no reports of excessive bleeding, pain or infection with the advanced protocol.

Conclusion: Using the advanced protocol, WOCNs detected PU severity, assessed PUs, and treated PUs safely. This protocol prevented PU deterioration and/or facilitated wound healing.

Relevance to clinical practice: With proper training, WOCNs can assess and treat PUs safer and quicker than when a physician's assessment is required, leading to an improvement in wound healing and prevention of PU deterioration.

Keywords: pressure ulcers, WOCN, interventions, protocols, deep tissue injury

\section{Introduction}

Pressure ulcers (PUs), also commonly called bedsores, are caused by pressure or by pressure in combination with shear. ${ }^{1}$ Once a PU becomes severe, it is likely to progress to a chronic, non-healing PU. Although there are several staging systems, such as those by Shea, ${ }^{2}$ the National Pressure Ulcer Advisory Panel, ${ }^{3}$ and the Japanese Society of Pressure Ulcers, ${ }^{4}$ the depth of PUs and damage to deep tissue are only classified by visual inspection. In general, severe PUs are defined as those causing full-thickness skin loss and categorized as stage III, IV (Table 1). 
Takeda et $\mathrm{al}^{5}$ reported that $39.6 \%$ of PUs in Japan are described as severe, which is considerably higher than the rate of $14 \%$ reported in the United States. ${ }^{6}$ Thus, the high prevalence of severe PUs is an important issue requiring attention. Some factors implicated in PU deterioration include general health of the patient, inappropriate pressure redistribution, and insufficient skin care techniques. ${ }^{7-9}$ Wound, ostomy, and continence nurses (WOCNs) are experts in the care of patients with PUs. They have specialized knowledge and expertise in reducing the prevalence and severity of PUs through methodologies such as pressure redistribution, skin care, wound care, and improved nutrition. Therefore, PU deterioration can be prevented by specialist nursing care in a timely manner.

However, at present, pathological examinations focus on deep tissue injury (DTI) caused by PU deterioration. The muscle is considered to be more susceptible to tissue damage from mechanical loading and oxygen deprivation ${ }^{10}$ than the dermis. Consequently, injury can be induced in the deep tissue and progress outward (bottom-up theory); ${ }^{11}$ this type of pressure-related injury to the deep tissue under the intact skin is defined as DTI. ${ }^{3}$ In general, these types of PUs are difficult to detect by visual inspection of the skin. On the other hand, critical colonization, which occurs when the bacterial burden in the wound bed increases in

Table I NPUAP staging system

\begin{tabular}{ll}
\hline Stage & Definition/descriptors \\
\hline Stage I & $\begin{array}{l}\text { Intact skin with non-blanchable redness of a } \\
\text { localized area usually over a bony prominence. } \\
\text { Darkly pigmented skin may not have visible } \\
\text { blanching; its color may differ from the } \\
\text { surrounding area. } \\
\text { Partial-thickness loss of dermis presenting as a } \\
\text { shallow open ulcer with a red pink wound bed, } \\
\text { without slough. May also present as an intact } \\
\text { or open/ruptured serum-filled blister. } \\
\text { Full-thickness tissue loss. Subcutaneous fat may } \\
\text { be visible but bone, tendon, or muscle are not } \\
\text { exposed. Slough may be present but does not } \\
\text { obscure the depth of tissue loss. May include } \\
\text { undermining and tunneling. } \\
\text { Full-thickness tissue loss with exposed bone, } \\
\text { tendon, or muscle. Slough or eschar may be } \\
\text { present on some parts of the wound bed. } \\
\text { Often includes undermining and tunneling. } \\
\text { Purple or maroon localized area of discolored } \\
\text { intact skin or blood-filled blister due to damage } \\
\text { tissue injury - depth } \\
\text { unknown }\end{array} \quad \begin{array}{l}\text { of underlying soft tissue from pressure and/or } \\
\text { shear. The area may be preceded by tissue } \\
\text { that is painful, firm, mushy, boggy, warmer, or } \\
\text { cooler as compared to adjacent tissue. }\end{array}$ \\
\hline
\end{tabular}

Abbreviation: NPUAP, National Pressure Ulcer Advisory Panel. association with a host immune response, is a relatively new concept. ${ }^{12}$ Critical colonization may be associated with PU deterioration. There is increasing recognition of the concept of critical colonization, where wound healing may be delayed in the absence of the typical clinical features of infection. Therefore, the effectiveness of PU interventions is enhanced when DTI or critical colonization is detected and addressed early.

\section{Background}

Diagnosis of DTI includes observation as a primary component according to the National Pressure Ulcer Advisory Panel PU staging guidelines. The Japanese PU guidelines ${ }^{13}$ recommend diagnosing DTI by palpation for induration or bogginess, determining changes in skin temperature, ${ }^{3}$ and ultrasonography (US) of the deep tissue..$^{14}$ US offers several advantages, such as no risk of radiation exposure and less pain; thus, it can be easily repeated in follow-up observations and can be performed in real-time at the bedside. A previous cohort study reported that the use of high-frequency US can help visualize the deep tissue to assess the degree of damage. ${ }^{15}$ Aoi et al reported the US characteristics that could predict deterioration. ${ }^{15}$

Thermography has been tested to detect latent inflammation in PUs, including those with critical colonization. ${ }^{16}$ Nakagami et al reported that PUs were classified into two groups depending on whether the wound site temperature was lower or higher than the peri-wound skin temperature; a higher wound site temperature may imply the presence of critical colonization. ${ }^{16}$ However, international studies on the mechanisms and effects of critical colonization are only in the early stages, and no interventional studies have focused on DTI and critical colonization with the aim of preventing PU deterioration or providing systematic protocols for the management of PUs.

Part of the management of PUs includes wound bed preparation (WBP) to promote the wound healing process. ${ }^{17}$ To address excess exudate, during WBP, the necrotic tissue is removed by debridement, followed by the application of appropriate dressings or negative pressure wound therapy (NPWT). In Japan, only physicians can legally perform WBP using these methods; however, any delay in therapy can lead to more severe PUs and delayed healing.

A systematic protocol is required that allows nurses to undertake appropriate assessment and intervention for addressing DTI and critical colonization as early as possible. This protocol must be safe and must involve non-invasive assessment techniques and minimally invasive treatments. 
In the present study, we created a protocol to meet this requirement.

Based on results from a previous study, it is thought that low-frequency vibration promotes skin blood flow. ${ }^{18}$ In addition, the effects and safety of vibration have been tested in a preclinical study with human volunteers. ${ }^{19}$ In clinical studies, vibration therapy facilitated the healing of stage I PUs ${ }^{20}$ and stage III, IV PUs with the necrotic tissue. ${ }^{21}$ These results suggest that vibration therapy is an easy and safe therapeutic option in nursing that can accelerate healing. Therefore, this non-invasive adjunctive treatment may be useful for the management of PUs by WOCNs.

The purpose of this study was to investigate the effectiveness and safety of an advanced protocol independently followed by trained WOCNs to prevent PU deterioration and accelerate healing in comparison with those of a conventional protocol also followed by WOCNs but under the direction of physicians.

\section{Methods \\ Design}

This was a prospective, non-randomized controlled trial to investigate the effectiveness of an advanced PU management protocol by comparing the severity of PUs and healing in two groups: an intervention group treated by trained WOCNs following an advanced PU management protocol, and a conventional protocol group treated by WOCNs following a conventional protocol. This protocol included six steps: 1) US to assess the deep tissue; 2) use of a non-contact thermometer (ST-717; Sukara Corp, Tokyo, Japan) to detect critical colonization; 3) conservative sharp debridement; 4) dressing selection; 5) NPWT, and 6) vibration therapy (RelaWave; Matsuda Micronics Corp, Chiba, Japan) as an adjunct treatment to improve tissue microcirculation. It was difficult to assemble enough facilities to implement the intervention because of legal issues with regard to it being an advanced PU management protocol; hence, random allocation was not possible.

The DESIGN-R validated PU status assessment tool was used to evaluate these two variables. ${ }^{4,22}$ The tool includes assessment of the depth, exudate, size, infection/inflammation, granulation tissue, necrotic tissue, and pocketing. The total score is calculated from six items, excluding the depth, and ranges from 0 to 66 points, with higher scores representing more severe PUs. The inter-rater reliability and predictive validity for wound healing using this scale have been established previously. ${ }^{22,23}$ The conventional protocol group (control group) received standard of care on the basis of PU prevention and topical treatment guidelines issued by the Japanese Society of Pressure Ulcers. ${ }^{13}$

\section{Participants}

We recruited ten WOCNs registered with the Japanese Society of Wound, Ostomy, and Continence Management to follow the advanced protocol as well as 20 WOCNs to serve as controls. All the nurses had at least 3 years of WOC (wound, ostomy, and continence) experience and had managed PU care in a hospital setting; the nurses were recruited from all across Japan. We used an optimal matching ratio of 2:1 for WOCNs because this was an observational study in which we could not control the randomization of the advanced protocol and control patient groups. Thus, the $2: 1$ ratio would result in a higher statistical power for the study than the 1:1 ratio. One of the original 20 WOCNs dropped out of the study before data collection; therefore, 19 WOCNs served as controls, thereby reducing the ratio to less than 2:1 (since ten nurses followed the advanced protocol) and affecting the number of patients in the advanced protocol group versus (vs) the conventional protocol group (123 vs 189). The two WOCN groups were matched in terms of years of WOC experience and the number of beds and type of hospital in which they worked.

WOCNs participating in the present study followed a series of PU prevention and topical treatment guidelines issued by the Japanese Society of Pressure Ulcers (2009). These guidelines are similar to those of the National Pressure Ulcer Advisory Panel. ${ }^{24}$

Patients who had at least one PU at any stage were recruited by WOCNs in each participating hospital. The patients were excluded if they were about to be discharged, their ulcer healed within 1 week. Also, the patients were excluded if their life expectancy was below 3 months because the goal of PU management of terminal care was different. If a patient had multiple ulcers, the most severe ulcer, defined as that with the highest DESIGN-R total score, was chosen for inclusion in the study. All the patients were admitted to facilities from July 2009 to December 2009. The patients participating in the present study were elderly, with no minor children.

\section{Sample size determination}

Based on a previous study, ${ }^{25}$ to detect a 3-point (standard deviation [SD] 7.72) clinically significant difference in the DESIGN-R ${ }^{4,22}$ score over 3 weeks with alpha $=0.05$, 100 subjects were required in each group for achieving a power of $80 \%$ and detecting statistical significance for 
intergroup differences in DESIGN-R scores. We exceeded the required statistically significant sample size and successfully recruited a total of 312 patients (ie, 123 patients in the advanced protocol group vs 189 in the conventional protocol group) as study participants.

\section{Intervention}

\section{Protocol}

Based on a literature review, an advanced PU management protocol was created to prevent wound deterioration and facilitate healing. As mentioned above, this protocol included six steps, and these steps were implemented for 3 weeks depending on the wound status.

The difference between the advanced protocol and the conventional protocol (control group) was that WOCNs in the advanced protocol group were trained to determine which of the six techniques/steps in the protocol could be used and were then permitted to perform these interventions without a physician's assessment. WOCNs in the conventional protocol (control group) were not permitted to use these techniques without a physician's assessment and permission because this is the standard of care in Japan.

In the conventional protocol group, according to the standard of care, debridement was solely performed by physicians, only if they noted a need for debridement. In the advanced protocol group, both physicians and WOCNs performed debridement and WOCNs were permitted to perform dressing selection and NPWT.

\section{Training in advanced PU management}

Training in advanced PU management for WOCNs using the advanced protocol was conducted by a nurse practitioner from the United States, expert nurses and physicians from March 6, 2009 to March 8, 2009 and May 26, 2009 that included lectures, skill demonstration, practice, and group discussion. The lecture component lasted for 45 hours, with various lectures being held by nurses, physicians, and radiologists who underwent training through our program; the lecture-based instruction was followed by 60 hours of onsite clinical experience, with physicians at each facility leading these efforts. WOCNs were instructed to assess the need for and implementation of the six steps as follows:

1. US: palpation for induration or bogginess; WOCNs following the advanced protocol were also trained to interpret the US findings on the basis of four types of abnormal signs: unclear layered structure, hypoechoic lesion, discontinuous fascia, and heterogeneous hypoechoic area. These signs were representative of DTI. ${ }^{15}$
2. Non-contact thermometer: temperature in a non-necrotic wound, peri-wound skin and wound edge was taken weekly. A higher temperature in the peri-wound skin compared with that in the wound may indicate critical colonization. ${ }^{16}$ This technique was applied for all patients.

3. Training in conservative sharp debridement based on the necrotic score ( $\mathrm{N}$ score) of the DESIGN-R tool.

4. Training in dressing selection based on Japanese Society of Pressure Ulcers (2009) guidelines.

5. Training in NPWT, applied for stage III or IV non-necrotic wounds.

6. Training in vibration therapy, applied for all patients.

\section{Data collection}

Each group of WOCNs was provided with instructions on data collection and completion of the questionnaire (Tables 2-4).

A researcher and a plastic surgeon, both blinded to the two protocol groups, determined the DESIGN-R score on the basis of photographs taken during the initial intervention and 1, 2, and 3 weeks later, without evaluating the exudate amount, wound size and pocketing (undermining). The intraclass correlation coefficient was 0.851 . Because it was not possible to evaluate the exudate amount on the basis of the photographs, these data were directly recorded by WOCNs.

Table 2 Characteristics of the hospitals and WOCNs

\begin{tabular}{|c|c|c|c|}
\hline & $\begin{array}{l}\text { Intervention } \\
\text { group } \\
n=10\end{array}$ & $\begin{array}{l}\text { Control } \\
\text { group } \\
n=19\end{array}$ & $P$-value \\
\hline Type of hospital n (\%) & & & $0.492^{\mathrm{b}}$ \\
\hline Community hospital & $2(20.0)$ & $8(42.1)$ & \\
\hline Long-term care & $2(20.0)$ & $2(10.5)$ & \\
\hline University hospital & $6(60.0)$ & $9(47.4)$ & \\
\hline $\begin{array}{l}\text { Number of beds, } \\
\text { mean (SD) }\end{array}$ & $747.2(260.2)$ & $718.9(253.2)$ & $0.946^{\mathrm{a}}$ \\
\hline RN staff/patient ratio & $0.82(0.26)$ & $0.87(0.12)$ & $0.640^{\mathrm{a}}$ \\
\hline $\begin{array}{l}\text { The number of WOCNs } \\
\text { at the facility }\end{array}$ & $2.2(0.6)$ & $2.0(1.0)$ & $0.542^{\mathrm{a}}$ \\
\hline Nursing experience & $18.0(4.9)$ & $16.6(5.5)$ & $0.330^{\mathrm{a}}$ \\
\hline WOC care experience & $7.4(3.3)$ & $5.6(2.4)$ & $0.195^{\mathrm{a}}$ \\
\hline \multicolumn{3}{|c|}{ Educational background n (\%) } & $0.162^{b}$ \\
\hline $\begin{array}{l}\text { Nursing School } \\
\text { (3 years) }\end{array}$ & $6(60.0)$ & 14 (73.7) & \\
\hline \multicolumn{4}{|l|}{ College (3 years) } \\
\hline $\begin{array}{l}\text { Nursing University } \\
\text { (4 years) }\end{array}$ & 0 & 0 & \\
\hline $\begin{array}{l}\text { Master of Science } \\
\text { in Nursing }\end{array}$ & $3(30.0)$ & $2(10.5)$ & \\
\hline Doctor of Philosophy & I (I0.0) & 0 & \\
\hline
\end{tabular}

Notes: aMann-Whitney $U$ test; 'Fisher's exact test.

Abbreviations: WOCNs, wound, ostomy, and continence nurses; SD, standard deviation; RN, registered nurse; WOC, wound, ostomy, and continence. 
Table 3 Comparison of patient characteristics

\begin{tabular}{|c|c|c|c|c|c|}
\hline & $\mathbf{n}$ & $\begin{array}{l}\text { Intervention } \\
\text { group }(n=123)\end{array}$ & $\mathbf{n}$ & $\begin{array}{l}\text { Control } \\
\text { group }(n=189)\end{array}$ & $P$-value \\
\hline Age, mean (SD) & 123 & $77(16)$ & 189 & $74.5(14.1)$ & $0.235^{\mathrm{a}}$ \\
\hline Sex, n (\%) & 123 & & 189 & & $0.327^{\mathrm{b}}$ \\
\hline Male & & $64(52.0)$ & & $109(57.7)$ & \\
\hline Female & & $59(48.0)$ & & $80(42.3)$ & \\
\hline The number of ulcers, mean (SD) & 123 & $\mathrm{I} .8(\mathrm{I} .4)$ & 189 & $\mathrm{I} .4(\mathrm{I} .0)$ & $0.010^{\mathrm{a}}$ \\
\hline Serum albumin level (g/dL), mean (SD) & 115 & $2.7(0.6)$ & 180 & $2.7(0.7)$ & $0.634^{\mathrm{a}}$ \\
\hline Hemoglobin level (g/dL), mean (SD) & 121 & $10.5(2.1)$ & 187 & $10.3(2.1)$ & $0.479^{a}$ \\
\hline Serum C-reactive protein level (mg/dL), mean (SD) & 122 & $6.5(7.3)$ & 183 & $7.2(7.6)$ & $0.255^{\mathrm{a}}$ \\
\hline Braden score, mean (SD) & 123 & II (3.2) & 189 & $12.3(3.2)$ & $0.018^{a}$ \\
\hline \multicolumn{6}{|l|}{ Diagnosis, n (\%) } \\
\hline Cerebrovascular & 123 & $46(37.4)$ & 189 & $53(28.0)$ & $0.105^{\mathrm{b}}$ \\
\hline Bone & 123 & I4 (I I.4) & 189 & $17(9.0)$ & $0.562^{\mathrm{b}}$ \\
\hline Cancer & 123 & $22(17.9)$ & 189 & $37(19.6)$ & $0.768^{\mathrm{b}}$ \\
\hline Infection & 123 & $44(35.8)$ & 189 & $59(31.2)$ & $0.460^{\mathrm{b}}$ \\
\hline Dementia & 123 & $15(12.2)$ & 189 & $12(6.4)$ & $0.098^{\mathrm{b}}$ \\
\hline Hypertension & 123 & $23(18.7)$ & 189 & $19(10.1)$ & $0.04 I^{\mathrm{b}}$ \\
\hline Diabetes & 123 & $27(22.0)$ & 189 & $44(23.3)$ & $0.890^{\mathrm{b}}$ \\
\hline Renal failure & 123 & $9(7.3)$ & 189 & $19(10.0)$ & $0.544^{b}$ \\
\hline Electrolyte disorder & 123 & $12(9.8)$ & 189 & II (5.8) & $0.267^{b}$ \\
\hline Others & 123 & $49(39.8)$ & 189 & $57(30.2)$ & $0.087^{b}$ \\
\hline Pressure ulcer site, $\mathrm{n}(\%)$ & & & & & $0.828^{\mathrm{b}}$ \\
\hline Sacrum & & $55(44.7)$ & & $78(4 \mid .3)$ & \\
\hline Coccygeal & & $21(17.1)$ & & $40(21.1)$ & \\
\hline Greater trochanter & & $12(9.8)$ & & $19(10.1)$ & \\
\hline Heel & & $7(5.7)$ & & $6(3.2)$ & \\
\hline Spine & & $3(2.4)$ & & $6(3.2)$ & \\
\hline Others & & $25(20.3)$ & & $40(21.1)$ & \\
\hline Pressure ulcer depth, n (\%) & 123 & & 189 & & $0.455^{\mathrm{b}}$ \\
\hline Stage I & & $5(4.0)$ & & $4(2.2)$ & \\
\hline Stage II & & $39(31.7)$ & & $67(35.4)$ & \\
\hline Stage III & & $28(22.8)$ & & $32(16.9)$ & \\
\hline Stage IV & & $16(13.0)$ & & $21(11.1)$ & \\
\hline Unstageable & & $35(28.5)$ & & $65(34.4)$ & \\
\hline Type of pressure-redistributing mattress, $\mathrm{n}(\%)$ & 123 & & 188 & & $<0.00 \mathrm{I}^{\mathrm{C}}$ \\
\hline Alternating pressure air mattress & & $42(34.2)$ & & $38(20.2)$ & \\
\hline Foam mattress & & $20(16.2)$ & & $69(36.7)$ & \\
\hline Low air pressure relief mattress (alternating air mattress) & & $59(48.0)$ & & $74(3.7)$ & \\
\hline Others & & $2(1.6)$ & & $7(39.4)$ & \\
\hline
\end{tabular}

Notes: aStudent's t-test; 'Pearson's chi-squared test; 'Fisher's exact test. Table is a comparison of the intervention and control groups. Pressure ulcer depth categorized using National Pressure Ulcer Advisory Panel staging system.

Abbreviation: SD, standard deviation.

Of note, it has been reported that both wound size and pocketing were high intra-class correlation coefficient $(r=1){ }^{4}$ Thus, we evaluated both wound size and pocketing obtained from the clinical notes by WOCNs.

To assess the safety of the advanced protocol and conventional protocol, patients' physical discomfort was evaluated using the National Cancer Institute's Common Terminology Criteria for Adverse Events version 4.0 (2009). The inter-rater reliability and validity for Common Terminology Criteria for Adverse Events have been established previously. ${ }^{26-28}$ Physical discomfort was defined as excessive bleeding due to sharp debridement or NPWT, or pain or infection associated with these interventions.
The characteristics of the participating hospitals, WOCNs, patients and PUs were recorded for comparison (Table 2). Hospital characteristics included the type of hospital, number of beds, registered nursing staff/patient ratio, and number of WOCNs at the facility. WOCN characteristics included nursing experience, WOC experience, and educational background (Table 2). Patient demographics included age, sex, number of ulcers, serum albumin, hemoglobin, serum C-reactive protein, Braden score, ${ }^{29,30}$ and diagnosis (Table 3).

PU characteristics included the ulcer location (Table 3) and total DESIGN-R score (Table 4). The type of pressure redistribution mattress used in the hospital setting was also recorded (Table 3). 
Table 4 Pressure ulcer characteristics

\begin{tabular}{|c|c|c|c|c|c|c|c|}
\hline & \multicolumn{3}{|c|}{$\begin{array}{l}\text { Intervention } \\
\text { group }(n=\mid 23)\end{array}$} & \multicolumn{3}{|c|}{$\begin{array}{l}\text { Control } \\
\text { group }(n=\mid 89)\end{array}$} & \multirow[t]{2}{*}{$P$-value } \\
\hline & $n$ & Mean & $\overline{S D}$ & $n$ & Mean & SD & \\
\hline \multicolumn{8}{|l|}{ (baseline) } \\
\hline$D$ & & 3.6 & 1.7 & & 3.8 & 1.8 & 0.509 \\
\hline$E$ & & 2.1 & 1.6 & & 2.2 & 1.6 & 0.529 \\
\hline S & & 5.7 & 2.5 & & 6.1 & 2.8 & 0.186 \\
\hline I & & 0.4 & 1.0 & & 0.6 & 1.4 & 0.191 \\
\hline G & & 3.1 & 2.6 & & 3.2 & 2.6 & 0.816 \\
\hline $\mathrm{N}$ & & 2.0 & 2.2 & & 2.4 & 2.2 & 0.093 \\
\hline$P$ & & 2.1 & 5.1 & & 1.7 & 5.3 & 0.444 \\
\hline $\begin{array}{l}\text { Total score } \\
\text { (week I) }\end{array}$ & 123 & 12.8 & 10.4 & 189 & 15.2 & 12.0 & 0.070 \\
\hline $\mathrm{D}$ & & 3.0 & 2.0 & & 3.2 & 1.9 & 0.549 \\
\hline $\mathrm{E}$ & & 1.8 & 1.6 & & 2.1 & 1.7 & 0.100 \\
\hline S & & 4.4 & 3.1 & & 5.1 & 3.1 & 0.044 \\
\hline I & & 0.1 & 0.5 & & 0.4 & 0.9 & 0.008 \\
\hline G & & 2.5 & 2.5 & & 3.0 & 2.5 & 0.090 \\
\hline $\mathrm{N}$ & & 1.7 & 2.0 & & 2.4 & 2.1 & 0.004 \\
\hline$P$ & & 2.3 & 5.1 & & 2.3 & 6.4 & 0.936 \\
\hline $\begin{array}{l}\text { Total score } \\
\text { (week 2) }\end{array}$ & 105 & 11.4 & 10.6 & 165 & 13.8 & 12.9 & 0.098 \\
\hline $\mathrm{D}$ & & 2.6 & 2.0 & & 2.7 & 1.9 & 0.624 \\
\hline E & & 1.6 & 1.6 & & 1.9 & 1.8 & 0.108 \\
\hline$S$ & & 3.7 & 3.0 & & 4.4 & 3.4 & 0.083 \\
\hline I & & 0.1 & 0.4 & & 0.3 & 0.7 & 0.005 \\
\hline G & & 2.3 & 2.4 & & 2.5 & 2.5 & 0.426 \\
\hline $\mathrm{N}$ & & 1.4 & 1.9 & & 2.1 & 2.0 & 0.006 \\
\hline$P$ & & 2.3 & 5.0 & & 2.6 & 6.5 & 0.733 \\
\hline $\begin{array}{l}\text { Total score } \\
\text { (week 3) }\end{array}$ & 99 & 10.6 & 10.8 & 149 & 12.2 & 13.2 & 0.318 \\
\hline $\mathrm{D}$ & & 2.4 & 1.9 & & 2.6 & 2.0 & 0.612 \\
\hline E & & 1.6 & 1.5 & & 1.9 & 1.9 & 0.231 \\
\hline S & & 3.5 & 3.1 & & 4.1 & 3.4 & 0.196 \\
\hline I & & 0.1 & 0.3 & & 0.2 & 0.9 & 0.115 \\
\hline G & & 2.1 & 2.4 & & 2.5 & 2.4 & 0.247 \\
\hline $\mathrm{N}$ & & 1.4 & 1.7 & & 1.9 & 1.9 & 0.022 \\
\hline$P$ & & 2.6 & 5.5 & & 2.9 & 7.0 & 0.719 \\
\hline \multicolumn{8}{|c|}{ Differences in scores } \\
\hline Week 0-I & 123 & 2.5 & 5.2 & 189 & 0.9 & 5.3 & 0.008 \\
\hline Week I-2 & 105 & 1.5 & 3.4 & 165 & 1.4 & 4.6 & 0.725 \\
\hline Week 2-3 & 98 & 0.8 & 2.5 & 148 & 0.7 & 4.5 & 0.831 \\
\hline Week 0-3 & 99 & 4.8 & 6.6 & 149 & 3.4 & 7.4 & 0.120 \\
\hline
\end{tabular}

Notes: Student's $t$-test. The total score is calculated from six items, excluding D (depth). E (exudate), S (size), I (infection/inflammation), G (granulation tissue), $\mathrm{N}$ (necrotic tissue), $\mathrm{P}$ (pocketing).

Abbreviation: SD, standard deviation.

\section{Calculation of the percentage of implementation of the advanced protocol techniques by WOCNs vs physicians}

We recorded the percentage of implementation of each of the six techniques in the advanced protocol by WOCNs. A researcher and a plastic surgeon involved in wound management determined which patients should undergo US for "suspected DTI" on the basis of photographs taken during the initial intervention. WOCNs used their training to determine which patients would undergo US.

A stage III or IV wound with no necrotic tissue is a common criterion for NPWT. PUs with an N score of 3 and 6 points of DESIGN-R (possible scores: $\mathrm{N}=0$ : none; 3 : soft necrotic tissue exists; 6: hard and thick necrotic tissue is attached to the wound) were assessed as those requiring debridement. A non-contact thermometer, vibration therapy, and dressings were used in all the patients, regardless of US findings or the DESIGN-R score. The percentage of implementation of each technique by WOCNs (vs physicians) in the advanced protocol group was calculated using the total number of patients who underwent each technique as the denominator, with the number who received the technique on the basis of an advanced protocol group WOCN's and physician's (vs physician's [control group]) assessment as the numerator.

\section{Ethical considerations}

The study was approved by the Research Ethics Committee of the University of Tokyo Graduate School of Medicine (number 2436) and each participating institution. All the participants received detailed information about the study, and directors of each institution provided written consent for WOCNs in the advanced protocol group to use the techniques. All the study participants in the two protocol groups provided written informed consent.

\section{Data analysis}

All analyses were conducted using SAS version 9.2 (SAS Institute Inc., Cary, NC, USA). $P$-values $\leq 0.05$ were considered statistically significant. Data were summarized as mean (SD). We used Pearson's chi-square test or Fisher's exact test as well as the Student's $t$-test or Mann-Whitney $U$ test to compare the groups.

Logistic regression analysis was used to determine the impact of the advanced protocol on PU deterioration as a measure of the effectiveness of the protocol. PU deterioration was defined as an increase in the total DESIGN-R score of at least 1 point or 0 for no change from before measurement.

A mixed-effects model was used to study the effect of the advanced PU protocol on changes in the severity of PU. The DESIGN-R score served as a dependent variable. A decrease of at least 1 point in the total DESIGN-R score was regarded as PU improvement. The random effect within patients was included for differentiating between-patients effect. The model was adjusted for confounding variables, including the nurses' skill, WOC care experience, number of beds and 
significant baseline variables. The interactions between the group and the time course of the dependent variable were tested (SAS MIXED procedure, covariance structure: compound symmetry). If the interaction was statistically significant, differences in the dependent variable at each week were tested by post hoc analyses, and the least-square means of the dependent variable with $95 \%$ confidence intervals were calculated each week by ESTIMATE statement.

\section{Results}

\section{Characteristics of hospitals and WOCNs}

Ten WOCNs of the participating facilities followed the advanced protocol, whereas 19 served as controls and followed a conventional protocol. The characteristics of the hospitals and WOCNs are shown in Table 2. There were no significant differences in basic characteristics between the two groups, including the mean number of years of experience.

\section{Overview of patients}

Patient details are shown in Figure 1. In total, 1,203 patients with PUs were hospitalized during the study period. Of these, 126 patients could have been included in the intervention group, but three were excluded; data from the remaining 123 patients were analyzed. In total, 199 patients could have been included in the control group, but ten were excluded; data from the remaining 189 patients were analyzed. The exclusion criteria were as follows: wound healed within 1 week; patient underwent surgery for PU, and terminal patients. Some patients declined to participate in the study.

Baseline information on the patients with PUs is shown in Table 3. The mean number of PUs per patient was $1.8(\mathrm{SD}=1.4)$ in the advanced protocol group and $1.4(\mathrm{SD}=1.0)$ in the control group, indicating that the patients in the advanced protocol group had more PUs $(P=0.010)$. The advanced protocol group had a baseline Braden score of $11.4(\mathrm{SD}=3.2)$ points, which was approximately 1 point lower than that in the control group (12.3 points [ $\mathrm{SD}=3.2]$ ). The advanced protocol group contained more patients who were at risk of developing PU $(P=0.018)$. There were no differences between the two groups in terms of sex, type of hospital, anatomical location of PU, or PU depth. Significant differences between the groups were found with regard to the type of pressure redistribution mattress used $(P<0.001)$ and the item "hypertensive disorders" $(P=0.041)$.

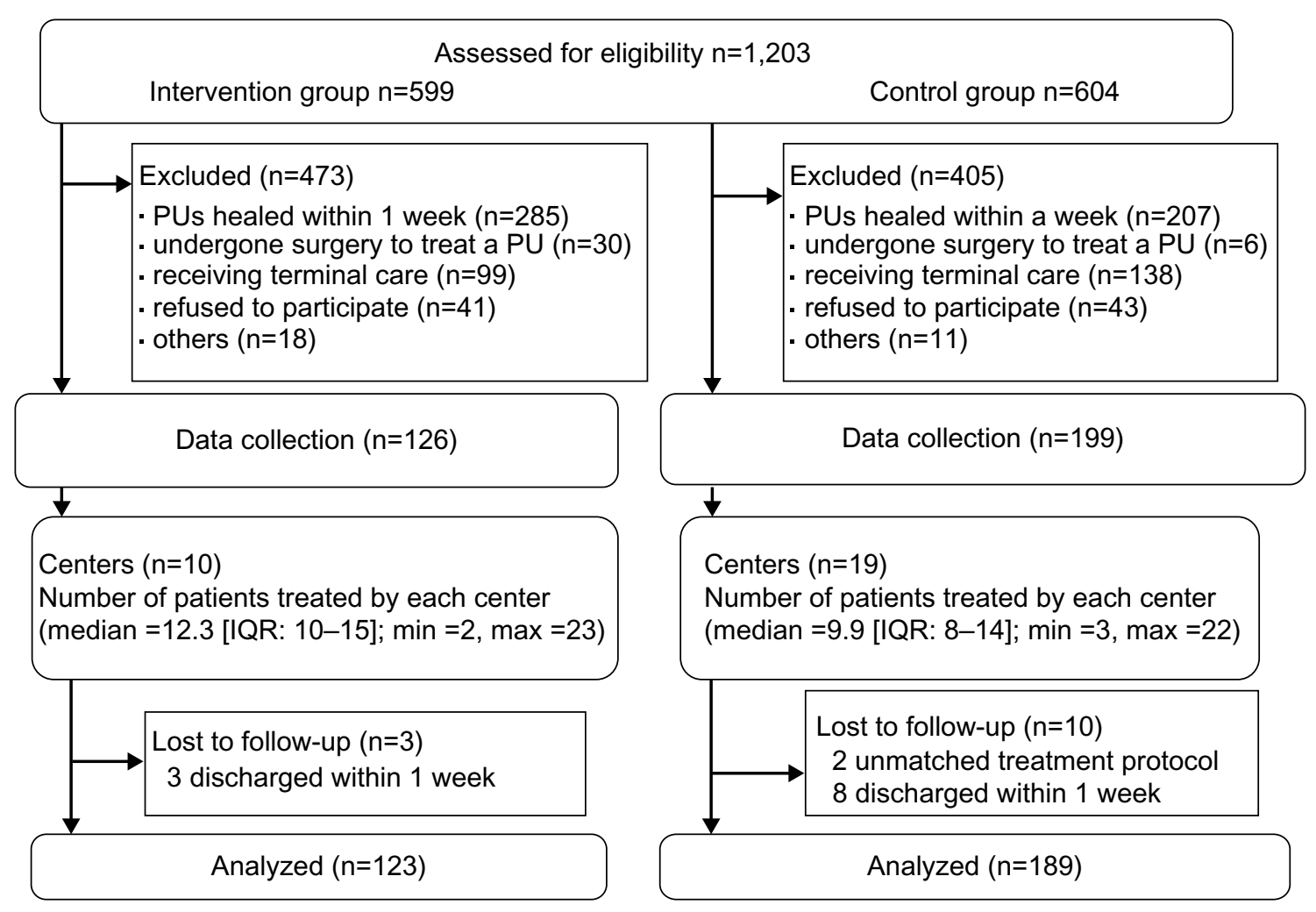

Figure I Patient details.

Abbreviations: IQR, interquartile range; max, maximum; min, minimum; PUs, pressure ulcers. 


\section{Characteristics of PUs}

The individual item DESIGN-R score and total score at baseline, changes in scores over time and differences in scores are shown in Table 4. There were no significant differences between the two protocol groups with regard to any specific DESIGN-R measurement or the total score at baseline. There was no significant difference in DESIGN-R score at the beginning and end of the observation period; however, a significant change $(P=0.008)$ was observed in week 1 , indicating accelerated healing of PUs in the advanced protocol group in week 1 .

\section{Percentage of implementation of the advanced PU management protocol}

The percentage of implementation of each of the six tools in the advanced protocol by WOCNs (independent of physicians) is shown in Figures 2 and 3.

When comparing the advanced protocol group with the control group, it should be noted that there were significant differences in individual techniques used in the two groups. Non-contact thermometer assessment of the wound was performed in $100 \%$ patients in the advanced protocol group and in none of the patients in the control group $(P<0.001)$. US was used in $76.5 \%$ patients in the advanced protocol group and only $5.6 \%$ patients in the control group $(P<0.001)$. As noted previously, a researcher and a plastic surgeon involved in wound management determined that 17 patients should undergo US for "suspected DTI" on the basis of photographs taken during the initial intervention. WOCNs decided to use US to detect DTI in a total of 13 cases. In the control group, a total of 18 cases in week 1 were determined as "suspected DTI" on the basis of photographs; one of these cases underwent US performed by a physician.

NPWT was used in $69.6 \%$ of the advanced protocol group patients and in none of the control group patients $(P=0.031)$. Vibration therapy was used in $27.6 \%$ of the advanced protocol group patients and in none of the control group patients $(P<0.001)$. Legally, WOCNs cannot select the type of dressing to be used in Japan; therefore, we expected that dressing selection would be physician-directed in the control group. However, because 18 of the 19 control WOCNs (94\%) selected the dressing despite being directed not to do so, this parameter could not be compared between the two groups. During weeks 1, 2, and 3, 70.3\%, 75.9\%, and $80.5 \%$, respectively, of the advanced protocol group patients underwent debridement by either a WOCN or a physician and $44.0 \%, 43.4 \%$, and $49.0 \%$, respectively, of the control group patients underwent debridement by a physician (WOCNs were not permitted to perform debridement in the control group) $(P<0.001)$. Based on their $\mathrm{N}$ scores, a total of 64 advanced protocol group patients in week 1 should have undergone debridement; WOCNs and physicians performed debridement in 45 patients. In the control group, a total of 116 patients in week 1 were determined to need debridement

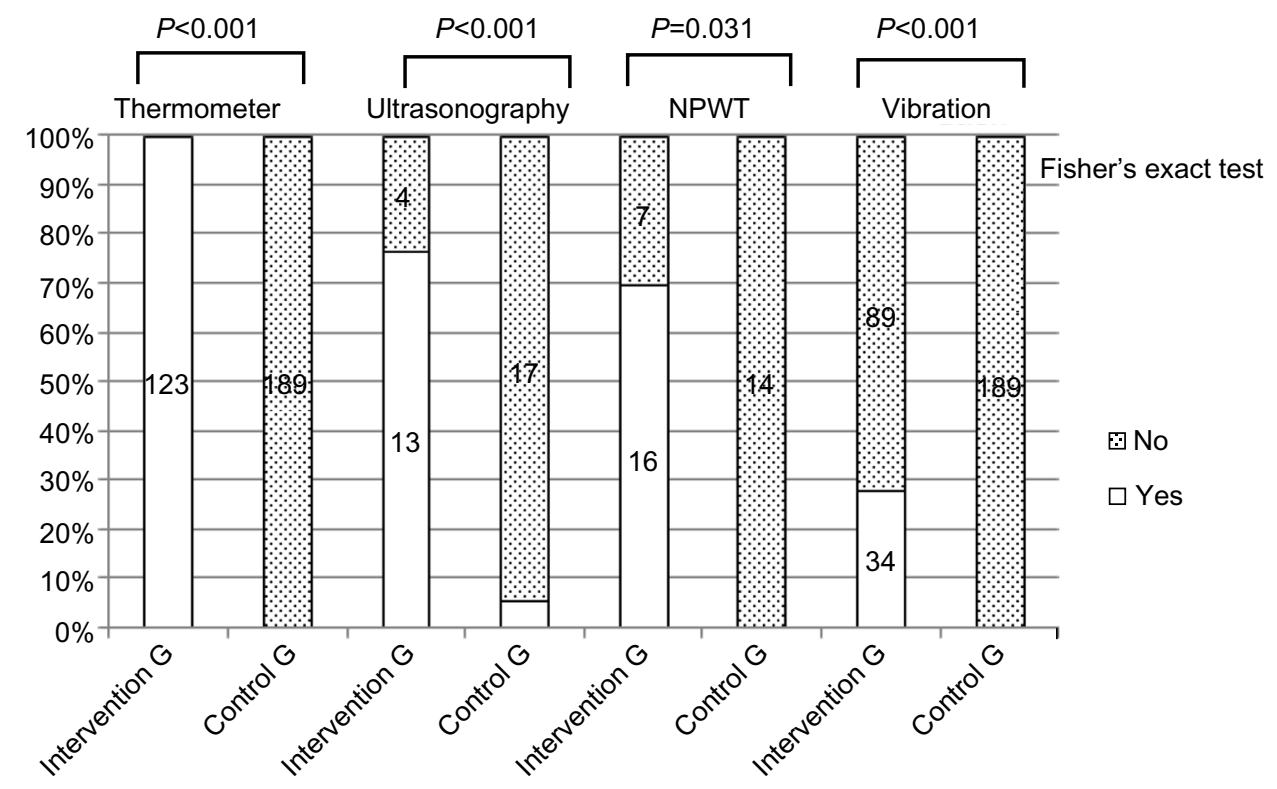

Figure 2 The percentage of implementation of the advanced pressure ulcer management protocol.

Notes: The percentage of implementation of each technique by WOCNs and physicians (vs physicians) in each group was calculated using the total number of patients who underwent each technique as the denominator, with the number receiving the technique based on an advanced-protocol WOCN's and physician's (vs physician's [conventional protocol]) assessment as the numerator. The numbers in the graph refer to the number of patients. The dotted area indicates the number of patients who did not undergo a given technique, whereas the white area shows the number of patients who underwent each technique.

Abbreviations: WOCNs, wound, ostomy, and continence nurses; vs, versus; G, group; NPWT, negative pressure wound therapy. 


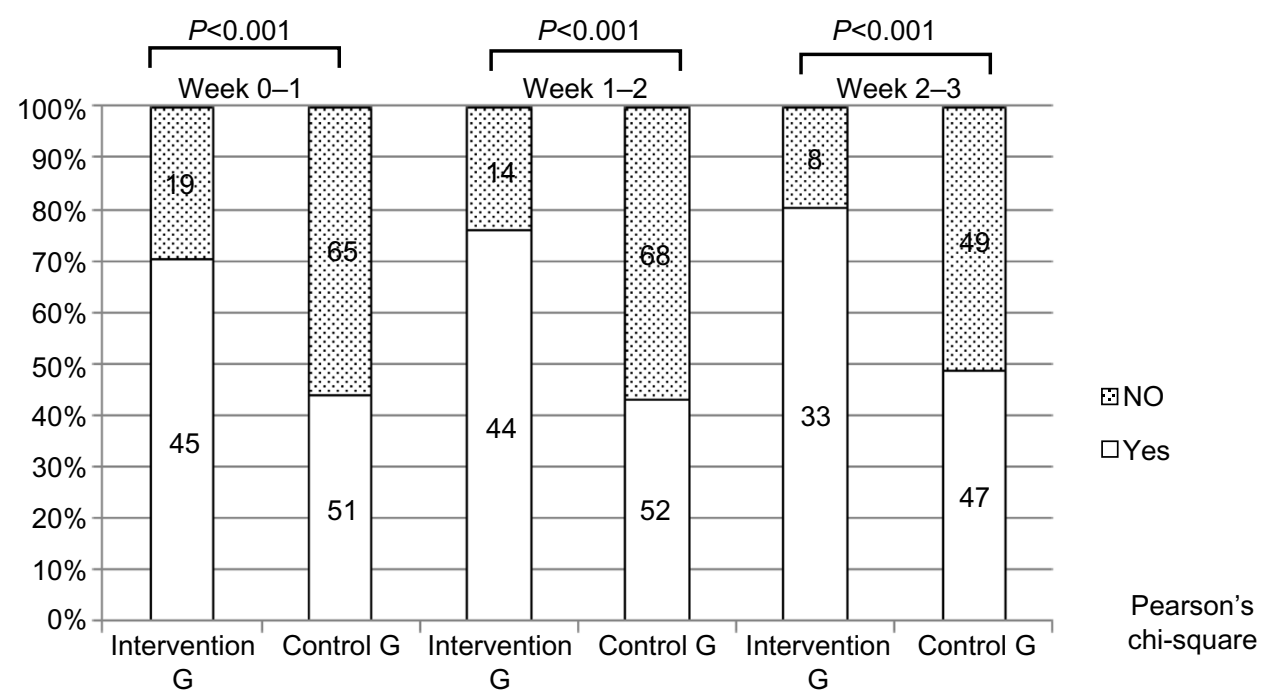

Figure 3 The percentage of implementation of debridement.

Notes: It was calculated using the total number of patients who were determined by $\mathrm{N}$ (necrotic) score to need debridement as the denominator each week. The number receiving the debridement technique based on an advanced-protocol WOCN's and physician's (vs physician's [conventional protocol]) assessment as the numerator.

Abbreviations: WOCNs, wound, ostomy, and continence nurses; vs, versus; G, group.

on the basis of the $\mathrm{N}$ score; 52 of these patients received debrided by a physician.

The $\mathrm{N}$ score and debridement characteristics were observed at weeks 2 and 3.

\section{Effect of advanced protocol on PU outcomes}

\section{Effect of intervention on PU deterioration}

To determine the effectiveness of the advanced protocol, a logistic regression model was used, with PU deterioration as a dependent variable between baseline and week 1. Adjusting for bias in individual nurses' skill and quality of care at a facility, the number of years of experience in WOC care, nursing experience, educational background, and number of beds were included. The number of PUs, type of pressure redistribution mattress, baseline Braden score, and condition (hypertension) had a significance of $P<0.05$ on univariate analysis and were entered into our calculations. Age and sex were entered as moderator variables. In week $0-1$, the number of patients with severe PU was 2.2 times higher in the control group than in the advanced protocol group (Table 5), and the advanced protocol was significantly associated with the prevention of PU deterioration (odds ratio $=2.24$, [ $95 \%$ confidence interval, 1.297-3.879], $P=0.004$ ) during the same time frame. No differences were observed in weeks $1-2$, weeks $2-3$, and weeks $0-3$.

\section{Effect of intervention on PU healing}

A mixed-effects model was used to study the influence of the advanced protocol on PU severity between baseline and week 3. Baseline variables, including the number of PUs, type of pressure redistribution mattress, baseline Braden score, and condition (hypertension), were adjusted as potential confounding variables. Age and sex were also included in the multivariate model. The results indicated that the interaction term for group and time was significant $(P=0.012)$, and a greater decrease in the DESIGN-R score was observed in the advanced protocol group than in the control group (Figure 4). The post hoc test revealed that least-square means of the total DESIGN-R score were significantly smaller in the advanced protocol group at 1,2 , and 3 weeks $(P=0.010$, 0.009 , and 0.015 , respectively), showing accelerated healing of PUs in the advanced protocol group.

\section{Patient safety}

There were no reports of excessive bleeding associated with sharp debridement or NPWT, and no pain or infection was associated with these interventions.

\section{Discussion}

The present prospective, non-randomized controlled trial revealed that using an advanced PU assessment and treatment protocol, with objective US and non-contact thermometer data, permitted patients to receive appropriate care tailored for the stage of their PUs, and that this early intervention prevented PU deterioration and accelerated healing. This is the first study in Japan using assessment techniques performed by WOCNs.

Prevention of PU deterioration depended on two issues. The first issue was ascertaining the extent of DTI to prevent the extension of DTI through appropriate pressure 
Table 5 Effect of intervention on pressure ulcer deterioration; logistic regression analyses between baseline and week I

\begin{tabular}{|c|c|c|c|c|c|c|c|c|c|}
\hline & B & SE & $\begin{array}{l}\text { Odds } \\
\text { ratio }\end{array}$ & $95 \% \mathrm{Cl}$ & $P$-value & $\begin{array}{l}\text { Hosmer and } \\
\text { Lemeshow test } \\
P \text {-value }\end{array}$ & $\chi^{2}$ & $\begin{array}{l}\text { Cox and } \\
\text { Snell }\end{array}$ & $\begin{array}{l}\text { Nagelkerke } \\
\mathbf{R}^{2}\end{array}$ \\
\hline $\begin{array}{l}\text { Advanced pressure } \\
\text { ulcer management } \\
\text { (no intervention) }\end{array}$ & 0.808 & 0.279 & 2.243 & $1.297-3.879$ & 0.004 & 0.868 & 3.878 & 0.084 & 0.112 \\
\hline WOC care experience & -0.002 & 0.006 & 0.998 & $0.987-1.009$ & 0.688 & & & & \\
\hline Nursing experience & 0.005 & 0.002 & 1.005 & $1.000-1.009$ & 0.054 & & & & \\
\hline \multicolumn{10}{|l|}{ Educational background (WOC) } \\
\hline $\begin{array}{l}\text { Nursing School vs } \\
\text { Graduate School }\end{array}$ & 0.404 & 0.961 & 1.497 & $0.696-3.220$ & 0.302 & & & & \\
\hline $\begin{array}{l}\text { Nursing College vs } \\
\text { Graduate School }\end{array}$ & 0.899 & 0.542 & 2.456 & $0.849-7.107$ & 0.097 & & & & \\
\hline Braden score (baseline) & 0.035 & 0.043 & 1.036 & $0.953-1.126$ & 0.404 & & & & \\
\hline The number of beds & 0 & 0.001 & 1.000 & $0.999-1.001$ & 0.897 & & & & \\
\hline The number of ulcers & 0.036 & 0.106 & 1.037 & $0.842-1.277$ & 0.736 & & & & \\
\hline \multicolumn{10}{|c|}{ Type of pressure-redistributing mattress } \\
\hline $\begin{array}{l}\text { Alternating pressure } \\
\text { air vs foam }\end{array}$ & 0.313 & 0.758 & 1.368 & $0.310-6.038$ & 0.679 & & & & \\
\hline Low air pressure vs foam & 0.153 & 0.754 & 1.165 & $0.266-5.104$ & 0.839 & & & & \\
\hline Others vs foam & -0.168 & 0.739 & 0.845 & $0.199-3.598$ & 0.820 & & & & \\
\hline Age & -0.017 & 0.009 & 0.983 & $0.965-1.002$ & 0.983 & & & & \\
\hline Sex (male) & 0.385 & 0.248 & 1.470 & $0.904-2.389$ & 0.120 & & & & \\
\hline Hypertension (no) & 0.142 & 0.369 & 1.152 & $0.560-2.373$ & 0.701 & & & & \\
\hline
\end{tabular}

Abbreviations: B, baseline; SE, standard error; $\mathrm{Cl}$, confidence interval; WOC, wound, ostomy, and continence; vs, versus.

redistribution and other preventive care, including frequency of turning and repositioning. Among the advanced protocol group, $76.5 \%$ patients underwent US, and there were significant differences between the groups with regard to the type of pressure redistribution mattress used. Performing US may have helped improve subsequent mattress selection and thereby prevented PU deterioration. Compared with the control group,

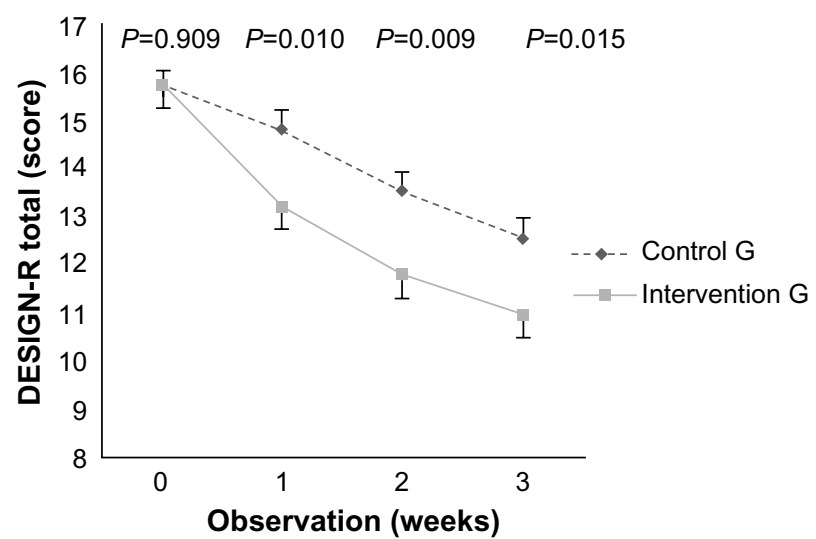

Figure 4 Effect of intervention on PU healing.

Notes: Change of least square means (LSMs) of the total DESIGN-R score over time. P-values were obtained weekly by post hoc analysis (Dunnett's method). LSMs and $95 \%$ confidence intervals, indicated by error bars, were calculated by the ESTIMATE statement. The interaction term for group and time was significant and a decrease in the DESIGN-R score was observed $(P=0.012, F=3.66)$ in the advanced protocol group.

Abbreviations: PU, pressure ulcer; G, group. more low-air pressure-relief mattresses, which are $15 \mathrm{~cm}$ thick alternating air mattresses, were used in the advanced group. However, the present study did not investigate a causal relationship in the qualitative study such as mattress selection after detected DTI; further studies are required to evaluate each of the six tools in the advanced protocol, including the evaluation of the successful implementation. The type of hospital and number of beds in the advanced group were matched with those in the control group; thus, there were not many differences in the mattress type between the two groups.

We found that in week 1, the risk of severe PUs was 2.2 times greater in the control group than in the advanced protocol group. The advanced PU management protocol was therefore effective in preventing PU deterioration at this early stage. Sato et $\mathrm{al}^{31}$ reported that stage I PUs took 3 weeks to develop and increase in severity. Early detection and intervention for DTI substantially reduces the time required for healing.

The second issue was that the advanced protocol group patients underwent debridement significantly more often than the conventional protocol group patients, suggesting that allowing WOCNs to proceed with assessment and treatment without physician orders led to timely treatment. Because it is widely accepted in wound care that wound debridement should be performed with appropriate timing, timely wound debridement is one of the quality indicators; wound 
debridement should be performed within 3 days after its clinical necessity has been documented. ${ }^{32}$ In the present study, less than $50 \%$ of the conventional protocol group patients underwent debridement by a physician. This result suggested that the target patients associated with debridement by WOCNs and physicians differed. Compared with physicians, WOCNs performed additional debridement in a higher number of patients, leading to a higher efficacy in the advanced protocol group. Further studies are required to evaluate of the process of debridement in each group.

Critical colonization leads to refractory PUs with delayed healing. In the past, there were no indices for detecting this condition, other than gross assessment. ${ }^{12}$ Clinically, critical colonization is suspected when healing is not apparent after 2 weeks, and treatment of infection is recommended. Assessment of the skin temperature using a non-contact thermometer in the advanced protocol group allowed early detection and intervention for critical colonization, resulting in accelerated healing. All the patients in the advanced protocol group were treated following this technique. Such non-invasive methods can be easily introduced into clinical practice because they can be readily performed at the bedside.

Biofilms are present in $60 \%$ of chronic wounds, ${ }^{33}$ reflecting critical colonization. In patients with a chronic leg ulcer, a biofilm reportedly re-forms 3 days after debridement, ${ }^{34}$ suggesting that repeated debridement is an effective way to eliminate biofilms in clinical settings. ${ }^{34}$ In the present study, debridement was performed more often by WOCNs, who removed biofilms that would have otherwise delayed the healing of chronic wounds.

According to the advanced PU management protocol, vibration therapy should be used for all patients; however, only $27.6 \%$ patients received vibration therapy. This result might be due to the difficulty of continuing use of the vibration device, because we educated only WOCNs in this study. Dissemination and implementation using vibration therapy without cooperation of the staff may be difficult. A lack of human resource development, including training of multidisciplinary team, would make the dissemination and implementation of technologies impossible in clinical practice. Thus, further study is needed.

There were no reports of complications associated with the interventions used in this study. The advanced protocol involved non-invasive assessment and minimally invasive intervention and focused on safety. The 105 hours training is required to implement the protocol, and this training ensured that the techniques were safe and effective. Therefore educating WOCNs in performing it is also important. It is necessary to investigate the effects of applying our findings in a clinical setting with regard to factors such as clinical effectiveness, patient safety, and cost-effectiveness. Therefore, we plan to investigate the cost of implementing the advanced protocol in future.

The participants in our study were elderly, with a mean age of 76.5 years. They were undernourished, had a mean Braden score of 11.4 points and were subject to compromised healing. However, an adequate clinical effect was achieved even in these at-risk patients. In low-risk patients, this protocol is expected to provide equal or better results.

The present study assumed that care would be provided by nurses and thus required the prior consent of all participating facilities. Randomization was deemed difficult, and the facilities were chosen by chance; therefore, there may have been a bias toward facilities with a clinical focus on PU care. The type of hospital, number of beds, and number of years of experience in WOC care may have affected the overall quality of care at a facility and the characteristics of its inpatients. However, WOCNs in the advanced protocol group were matched with control nurses to help address this issue. Bias was also considered during the study design to minimize its effects.

To avoid the occurrence of an observation bias, a researcher and a plastic surgeon, both blinded to the two protocol groups, determined the DESIGN-R score on the basis of photographs, without evaluating the exudate amount, wound size and pocketing. A previous study has reported the accurate assessment of wound appearance of PUs on photographs; ${ }^{35}$ thus, we used photographic data from digital camera images owing to blinding, which was a limitation of our study. In addition, we excluded terminal patients, patients who underwent surgery to treat PU, and patients whose PUs healed within 1 week; therefore, the findings should be generalized with caution.

\section{Conclusion}

Using our advanced protocol, WOCNs could detect PUs' state early and assess and treat PUs safely. This intervention permits patients to receive earlier preventive care and may prevent severe PU deterioration and accelerate healing.

\section{Relevance to clinical practice}

The care provided by WOCNs using our advanced PU protocol is safe and effective in accelerating healing and preventing PU deterioration; these findings are likely to have considerable impact on the medical care system in and outside Japan. With proper training, WOCNs can assess and treat PUs safely and quickly using their own judgment, without waiting for a physician's assessment; this early intervention by trained WOCNs helps decrease the incidence of severe PUs. 


\section{Acknowledgment}

This study was funded by a Health Labour Sciences Research Grant (2009-2010).

\section{Disclosure}

The authors declare they have no conflicts of interest.

\section{References}

1. National Pressure Ulcer Advisory Panel [homepage on the Internet]. Pressure Ulcer Category/Staging Illustrations; 2011. Available from: http://www.npuap.org/pr2.htm. Accessed January 3, 2015.

2. Shea JD. Pressure sores: classification and management. Clin Orthop Relat Res. 1975;(112):89-100.

3. Black J, Baharestani MM, Cuddigan J, et al. National Pressure Ulcer Advisory Panel's updated pressure ulcer staging system. Adv Skin Wound Care. 2007;20(5):269-274.

4. Sanada H, Moriguchi T, Miyachi Y, et al. Reliability and validity of DESIGN, a tool that classifies pressure ulcer severity and monitors healing. J Wound Care. 2004;13(1):13-18.

5. Takeda T, Shido K, Abe M, et al. Surveillance Committee Japanese Society of Pressure Ulcers, 2011. Surveillance Committee Report Jan JPU. 2010;13(4):625-632. Japanese.

6. VanGilder C, MacFarlane GD, Harrison P, Lachenbruch C, Meyer S. The demographics of suspected deep tissue injury in the United States: an analysis of the International Pressure Ulcer Prevalence Survey 2006-2009. Adv Skin Wound Care. 2010;23(6):254-261.

7. Cooper P, Gray D. Comparison of two skin care regimes for incontinence. Br J Nurs. 2001;10(Suppl 6):S6-S20.

8. Thompson P, Langemo D, Anderson J, Hanson D, Hunter S. Skin care protocols for pressure ulcers and incontinence in long-term care: a quasi-experimental study. Adv Skin Wound Care. 2005;18(8): $422-429$.

9. Clever K, Smith G, Bowser C, Monroe K. Evaluating the efficacy of a uniquely delivered skin protectant and its effect on the formation of sacral/buttock pressure ulcers. Ostomy Wound Manage. 2002;48(12): $60-67$.

10. Bouten CV, Oomens CW, Baaijens FP, Bader DL. The etiology of pressure ulcers: skin deep or muscle bound? Arch Phys Med Rehabil. 2003;84(4):616-619.

11. Doughty D, Ramundo J, Bonham P, et al. Issues and challenges in staging of pressure ulcers. J Wound Ostomy Continence Nurs. 2006;33(2): 125-130.

12. Sibbald RG, Woo K, Ayello EA. Increased bacterial burden and infection: the story of NERDS and STONES. Adv Skin Wound Care. 2006;19(8):447-461.

13. Japanese Society of Pressure Ulcers, editors. Guideline for Prevention and Management of Pressure Ulcers. Tokyo: Shourinsya Company; 2009. Japanese.

14. Nagase T, Koshima I, Maekawa T, et al. Ultrasonographic evaluation of an unusual peri-anal induration: a possible case of deep tissue injury. J Wound Care. 2007;16(8):365-367.

15. Aoi N, Yoshimura K, Kadono T, et al. Ultrasound assessment of deep tissue injury in pressure ulcers: possible prediction of pressure ulcer progression. Plast Reconstr Surg. 2009;124(2):540-550.

16. Nakagami G, Sanada H, Iizaka S, et al. Predicting delayed pressure ulcer healing using thermography: a prospective cohort study. $J$ Wound Care. 2010;19(11):465-466, 468, 470.
17. Schultz GS, Sibbald RG, Falanga V, et al. Wound bed preparation: a systematic approach to wound management. Wound Repair Regen. 2003;11 (Suppl 1):S1-S28.

18. Nakagami G, Sanada H, Matsui N, et al. Effect of vibration on skin blood flow in an in vivo microcirculatory model. Biosci Trends. 2007;1(3): 161-166.

19. Urasaki M, Sanada H, Tadaka M, et al. [Evaluation of the effect of vibration on blood flow in the calcaneal region]. Jpn J Pressure Ulcers. 2007;9(2):192-198. Japanese.

20. Arashi M, Sugama J, Sanada H, et al. Vibration therapy accelerates healing of Stage I pressure ulcers in older adult patients. Adv Skin Wound Care. 2010;23(7):321-327.

21. Ueda A, Sugama J, Okuwa M, et al. Effect of vibration on the healing of pressure ulcers with necrotic tissue. Jan JPU. 2010;12(2):111-117. Japanese.

22. Matsui Y, Furue M, Sanada H, et al. Development of the DESIGN-R with an observational study: an absolute evaluation tool for monitoring pressure ulcer wound healing. Wound Repair Regen. 2011;19(3): 309-315.

23. Sanada $\mathrm{H}$, Iizaka $\mathrm{S}$, Matsui $\mathrm{Y}$, et al. Clinical wound assessment using DESIGN-R total score can predict pressure ulcer healing Pooled analysis from two multicenter cohort studies. Wound Repair Regen. 2011;19(5):559-567.

24. European Pressure Ulcer Advisory Panel and National Pressure Ulcer Advisory Panel. Treatment of pressure ulcers: Quick Reference Guide. Washington DC: National Pressure Ulcer Advisory Panel; 2009.

25. Sanada H, Nakagami G, Mizokami Y, et al. Evaluating the effect of the new incentive system for high-risk pressure ulcer patients on wound healing and cost-effectiveness: a cohort study. Int J Nurs Stud. 2010;47(3):279-286.

26. Atkinson TM, Li Y, Coffey CW, et al. Reliability of adverse symptom event reporting by clinicians. Qual Life Res. 2012;21(7):1159-1164.

27. Patrick DL, Burke LB, Gwaltney CJ, et al. Content validity establishing and reporting the evidence in newly developed patientreported outcomes (PRO) instruments for medical product evaluation: ISPOR PRO Good Research Practices Task Force report: part 2 assessing respondent understanding. Value Health. 2011;14(8): 978-988.

28. Basch E, Abernethy AP, Reeve BB. Assuring the patient centeredness of patient-reported outcomes: content validity in medical product development and comparative effectiveness research. Value Health. 2011;14(8):965-966.

29. Bergstrom N, Braden B. A prospective study of pressure sore risk among institutionalized elderly. J Am Geriatr Soc. 1992;40(8):747-758.

30. Bergstrom N, Braden B, Boynton P, Bruch S. Using a research-based assessment scale in clinical practice. Nurs Clin North Am. 1995;30(3): 539-551.

31. Sato M, Sanada H, Konya C, Sugama J, Nakagami G. Prognosis of stage I pressure ulcers and related factors. Int Wound J. 2006;3(4):355-362.

32. Shekelle PG, MacLean CH, Morton SC, Wenger NS. Assessing care of vulnerable elders: methods for developing quality indicators. Ann Intern Med. 2001;135(8 Pt 2):647-652.

33. James GA, Swogger E, Wolcott R, et al. Biofilms in chronic wounds. Wound Repair Regen. 2008;16(1):37-44.

34. Wolcott RD, Rumbaugh KP, James G, et al. Biofilm maturity studies indicate sharp debridement opens a time-dependent therapeutic window. $J$ Wound Care. 2010;19(8):320-328.

35. Houghton PE, Kincaid CB, Campbell KE, Woodbury MG, Keast DH. Photographic assessment of the appearance of chronic pressure and leg ulcers. Ostomy Wound Manage. 2000;46(4):20-26, 28-30. 
Chronic Wound Care Management and Research

Dovepress

\section{Publish your work in this journal}

Chronic Wound Care Management and Research is an international, peer reviewed, open access, online journal publishing original research, reviews, editorials, and commentaries on the causes and management of chronic wounds and the major issues related to chronic wound management. Topics also include chronic wounds as comorbidities to other

conditions, patient adherence to therapy, and the economic burden of chronic wounds. The manuscript management system is completely online and includes a very quick and fair peer review system, which is all easy to use. Visit http://www.dovepress.com/testimonials.php to read real quotes from published authors.

Submit your manuscript here: http://www.dovepress.com/chronic-wound-care-management-and-research-journal 